\title{
COUNTEREXAMPLES TO QUASICONCAVITY FOR THE HEAT EQUATION
}

\author{
ALBERT CHAU AND BEN WEINKOVE
}

\begin{abstract}
We construct solutions to the heat equation on convex rings showing that quasiconcavity may not be preserved along the flow, even for smooth and subharmonic initial data.
\end{abstract}

\section{INTRODUCTION}

Let $\Omega_{0}$ and $\Omega_{1}$ be convex open sets with smooth boundary in $\mathbb{R}^{n}$ with $\bar{\Omega}_{1} \subset \Omega_{0}$. Assume that $\Omega_{1}$ contains the origin. Denote by $\Omega=\Omega_{0} \backslash \bar{\Omega}_{1}$ the open convex ring. We say that a function $u(x)$ on $\bar{\Omega}$ is quasiconcave if the sets

$$
\{x \in \bar{\Omega} \mid u(x) \geq c\} \cup \Omega_{1}
$$

are convex subsets of $\mathbb{R}^{n}$ for every $c \in \mathbb{R}$. Fix $T$ with $0<T \leq \infty$. A function $u=u(x, t)$ on $\bar{\Omega} \times[0, T)$ is called space-time quasiconcave if the sets

$$
\{(x, t) \in \bar{\Omega} \times[0, T) \mid u(x, t) \geq c\} \cup\left(\Omega_{1} \times[0, T)\right)
$$

are convex subsets of $\mathbb{R}^{n+1}$ for every $c \in \mathbb{R}$. Note that space-time quasiconcavity of $u(x, t)$ implies that $x \mapsto u(x, t)$ is quasiconcave on $\bar{\Omega}$ for each $t$.

It is a classical result that if $u$ is a harmonic function on $\Omega$ satisfying the Dirichlet boundary conditions

$$
\left.u\right|_{\partial \Omega_{0}}=0,\left.\quad u\right|_{\partial \Omega_{1}}=1
$$

then $u$ is quasiconcave [1, 17, 29. This result has been extended to solutions $u$ of more general elliptic PDEs, where there is a general principle that convexity properties of $\Omega_{0}$ and $\Omega_{1}$ imply convexity of the superlevel sets of $u$. These results are proved via "macroscopic" approaches involving functions of two points which could be far apart, or "microscopic" approaches using functions of the principal curvatures of the level sets together with constant rank theorems. See for example [2, 3, 4, 15, 7, 8, 9, 10, 11, 24, 25, [26, 30, 31, 32, 33] and the references therein. On the other hand, convexity properties fail for solutions to some elliptic PDEs [19, 34].

There has been considerable interest in parabolic versions of these classical results. Parabolic constant rank theorems in rather general contexts have been established by Hu-Ma 20, Chen-Hu 12 and Chen-Shi [13. An older result of Borell 6] assumes that the initial data is identically zero and shows that the solution $u(x, t)$ to the heat equation with boundary conditions (1.1) is space-time quasiconcave. This result has been extended to more general parabolic equations by Ishige-Salani [22, 23]. However,

Research supported in part by NSERC grant \#327637-06 and NSF grants DMS-1406164 and DMS1709544. 
the assumption of identically vanishing initial data is rather restrictive. This begs the question: what assumption on the initial data is necessary to ensure space-time quasiconcavity of the solution to the heat equation? Ishige-Salani 21 gave examples to show that quasiconcavity of the initial data is not sufficient. A natural condition considered in [14, 15] is that $u_{0}$ in addition be subharmonic (with sufficient regularity), namely $\Delta u_{0} \geq 0$. In this paper we provide a counterexample to show that this is still not sufficient to ensure quasiconcavity of the solution to the heat equation.

More precisely, we consider a classical solution $u$ of the following problem:

$$
\begin{cases}\partial u / \partial t=\Delta u, & \text { on } \Omega \times(0, T) \\ u(x, 0)=u_{0}(x), & x \in \Omega \\ u(x, t)=0, & (x, t) \in \partial \Omega_{0} \times[0, T) \\ u(x, t)=1, & (x, t) \in \partial \Omega_{1} \times[0, T),\end{cases}
$$

for $0<T \leq \infty$. Here $u_{0}$ is a smooth function on $\bar{\Omega}$ which satisfies the conditions

$$
\begin{aligned}
& u_{0}=1 \text { on } \partial \Omega_{1}, \quad u_{0}=0 \text { on } \partial \Omega_{0}, \quad x \cdot \nabla u_{0}(x) \leq 0 \text { on } \Omega \\
& \Delta u_{0} \geq 0 \text { on } \Omega \quad \text { but not identically zero. }
\end{aligned}
$$

We call such a function $u_{0}$ admissible.

We consider the following question: if an admissible $u_{0}$ is quasiconcave on $\bar{\Omega}$, does it follow that the solution $u(x, t)$ to (1.2) is space-time quasiconcave on $\bar{\Omega} \times[0, T)$ ? If not, is $x \mapsto u(x, t)$ quasiconcave on $\bar{\Omega}$ for each $t>0$ ?

We construct a counterexample to show that the answer to both of these questions is negative.

Theorem 1.1. For any $n \geq 2$, let $\Omega_{1}$ and $\Omega_{0}$ be balls in $\mathbb{R}^{n}$ centered at the origin, of radii 1 and 2 respectively, so that $\Omega$ is the annulus $1<r<2$. There is an admissible quasiconcave function $u_{0}$ with the following properties:

(i) The solution $u(x, t)$ to (1.2) is smooth on $\Omega \times(0, \infty)$ and continuous on $\bar{\Omega} \times$ $[0, \infty)$.

(ii) There exists $t_{0}>0$ such that the function $x \mapsto u\left(x, t_{0}\right)$ fails to be quasiconcave on $\bar{\Omega}$.

This example implies that the statement of [15, Theorem 3] (see the discussion in [22, Section 7]) requires additional hypotheses.

Our construction in Theorem 1.1 is based on the simple observation that the union of interiors of a sphere and a non-spherical ellipsoid is non-convex unless one is contained in the other. We use this observation as follows. We first find a radially symmetric admissible function $V$ which is close to 1 near the boundary of $\Omega_{1}$ and drops off rapidly to zero. For every positive time, the level sets of the heat flow solution starting from $V$ will then give a foliation of $\Omega$ by spheres. We then construct an admissible function $W$ whose level sets are spherical near the boundary of $\Omega_{1}$ but non-spherical ellipsoids as one goes outwards. We choose $u_{0}=(1-\varepsilon) V+\varepsilon W$, for $\varepsilon>0$ small, as initial data. The relatively large radially symmetric heat distribution of $(1-\varepsilon) V$ quickly emanates out and interacts with the ellipsoidal level sets of $\varepsilon W$ to give a non-convex superlevel set after some positive time. The proof of Theorem 1.1, given in Section 2, makes this heuristic argument precise. 
Note that by necessity our counterexample is not radially symmetric, and must have dimension $n>1$. If radial symmetry is imposed for $u_{0}$, which implies quasiconcavity of $x \mapsto u(x, t)$ for each $t$ (since the superlevel sets are balls in $\mathbb{R}^{n}$ ) it is natural to ask whether the stronger condition of space-time quasiconcavity follows. Our next counterexample shows that the answer to this is again negative for any $n \geq 1$.

Theorem 1.2. For any $n \geq 1$, let $\Omega_{1}$ and $\Omega_{0}$ be balls in $\mathbb{R}^{n}$ of radii $R$ and $R+1$ respectively, for a constant $R>1$. For $R$ sufficiently large, there is an admissible function $u_{0}$ on $\bar{\Omega}=\{R \leq r \leq R+1\} \subset \mathbb{R}^{n}$ with the following properties:

(i) $u_{0}$ is radially symmetric and hence if $u(x, t)$ solves (1.2) then $x \mapsto u(x, t)$ is quasiconcave on $\bar{\Omega}$ for every $t \geq 0$.

(ii) $u(x, t)$ is smooth on $\bar{\Omega} \times[0, \infty)$.

(iii) $u(x, t)$ is not space-time quasiconcave on $\bar{\Omega} \times[0, T)$ for any $T>0$.

In Theorem 1.2 any space-time level set $\partial \Omega_{c}:=\{(x, t) \in \bar{\Omega} \times[0, \infty) \mid u(x, t)=c\}$ for $c \in(0,1)$ will be given by a graph $t=f(|x|)$ where $f(r)$ is a smooth strictly increasing function defined on some interval $\left[r_{0}, r_{1}\right)$ where $f\left(r_{0}\right)=0$. In particular, $f$ is defined implicitly by $u(r, f(r))=c$ and differentiating this and using (1.2) gives

$$
f^{\prime \prime}(r)=\frac{-1}{u_{t}}\left(u_{r r}+2 u_{r t} f^{\prime}+u_{t t}\left(f^{\prime}\right)^{2}\right)
$$

We show that by solving an ordinary differential equation, we may choose the function $u_{0}$ so that the right hand side above is negative at $\left(r_{0}, 0\right)$, implying $f^{\prime \prime}\left(r_{0}\right)<0$ and thus $\partial \Omega_{c}$ is not convex. The details of this argument are given in Section 3 where we prove Theorem 1.2.

Finally, in Section 4 we give a different counterexample to space-time quasiconcavity using a "two-point function" as in [35] and inspired by the work of Rosay-Rudin [30]. It satisfies properties (i), (ii) of Theorem 1.2, but (iii) must be replaced by

(iii) $^{*} u(x, t)$ is not space-time quasiconcave on $\bar{\Omega}_{0} \times[0, T)$ for some $T>0$.

The argument using the two-point function is perhaps slightly more intuitive than that of Theorem 1.2 and the counterexample is defined on the annulus $\{1<r<2\}$.

Acknowledgements. The authors thank the referee for correcting some inaccuracies in a previous version of this paper.

\section{A COUNTEREXAMPle TO QUASICONCAVITY}

Let $\Omega_{0}, \Omega_{1}$ and $\Omega$ be as in the introduction. We first gather some well-known facts about solutions to (1.2).

Proposition 2.1. Let $u_{0}$ be an admissible function on $\bar{\Omega}$. Then there exists a unique continuous solution $u(x, t)$ to (1.2) on $\bar{\Omega} \times[0, \infty)$ which is smooth on $\Omega \times(0, \infty)$ and satisfies the following conditions for $(x, t) \in \Omega \times(0, \infty)$

(i) $0<u(x, t)<1$.

(ii) $u_{t}(x, t)=\Delta u(x, t)>0$.

(iii) $x \cdot \nabla u(x, t)<0$.

Moreover, as $t \rightarrow \infty, u(x, t)$ converges smoothly on $\Omega$ to the harmonic function $u_{\infty}$ with boundary conditions $\left.u_{\infty}\right|_{\partial \Omega_{0}}=0$ and $\left.u_{\infty}\right|_{\partial \Omega_{1}}=1$. 
Proof. The existence of a unique solution $u(x, t)$ to (1.2) with the stated regularity, and the convergence as $t \rightarrow \infty$ are classical, see for example [16. From (1.3) we have $0 \leq u_{0} \leq 1$ and then (i) follows from the strong maximum principle for parabolic equations. Parts (ii) and (iii) are proved in [15, Lemma 1] and are also consequences of the maximum principle.

We now start the proof of Theorem 1.1. Let $\Omega=\{1<r<2\}$ be as in the statement of the theorem and we assume for the rest of this section that $n \geq 2$. Part (i) of Theorem 1.1 is a consequence of the above proposition. For part (ii), we begin by defining two auxiliary functions $V_{\rho}$ and $W$.

Lemma 2.1. For any $\rho \in(1,3 / 2]$, define a radially symmetric function $V_{\rho}=V_{\rho}(r)$ on $\bar{\Omega}$ by

$$
V_{\rho}(r)= \begin{cases}\exp \left(\frac{n}{r-\rho}-\frac{n}{1-\rho}\right), & 1 \leq r<\rho \\ 0, & \rho \leq r \leq 2 .\end{cases}
$$

Then $V_{\rho}$ is an admissible function.

Proof. We drop the $\rho$ subscript. Observe that $V(r) \geq 0$ is smooth, decreasing on $[1,2]$, satisfies $V(1)=1$ and

$$
\begin{aligned}
(\Delta V)(r) & =V^{\prime \prime}(r)+\frac{(n-1) V^{\prime}(r)}{r} \\
& =n V(r)\left(\frac{2 r(r-\rho)+n r-(n-1)(r-\rho)^{2}}{r(r-\rho)^{4}}\right) \\
& \geq \frac{n}{4 r(r-\rho)^{4}} V(r) \geq 0
\end{aligned}
$$

where the second-to-last inequality follows from the inequalities $|r-\rho| \leq 1 / 2$ and $1 \leq r \leq 3 / 2$ when $r<\rho$.

We now use $V_{\rho}$ to define a non-radially symmetric function $W$.

Lemma 2.2. Fix $r_{0}, r_{1}$ with $1<r_{0}<r_{1} \leq 3 / 2$. There exists an admissible function $W$ on $\bar{\Omega}$ with the following properties.

(i) $W$ is radially symmetric on $1 \leq r \leq r_{0}$.

(ii) There exists a smooth strictly decreasing function $b$ on $\left[r_{0}, r_{1}\right]$ with $b\left(r_{0}\right)=1$ and $b\left(r_{1}\right) \in(0,1)$ such that if we define $E_{R}$ to be the ellipsoid with equation

$$
b(R)^{2} x_{1}^{2}+x_{2}^{2}+\cdots+x_{n}^{2}=R^{2}, \quad \text { for } R \in\left[r_{0}, r_{1}\right],
$$

then the level sets $\{W=c\}$ for $0<c<W\left(r_{0}\right)$ are the non-spherical ellipsoids $E_{R}$ for $R \in\left(r_{0}, r_{1}\right)$.

(iii) $W$ vanishes outside the ellipsoid $E_{r_{1}}$.

Proof. Let $V=V_{r_{1}}$ be as in Lemma 2.1 defined with $\rho=r_{1}$. Regarding $V$ as a function of $x_{1}, \ldots, x_{n}$ we compute for any $i, j=1, \ldots, n$ :

and

$$
V_{x_{i}}=-V(r) \frac{n x_{i}}{r\left(r-r_{1}\right)^{2}}
$$

$$
V_{x_{i} x_{j}}=V(r)\left(\frac{n^{2} x_{i} x_{j}}{r^{2}\left(r-r_{1}\right)^{4}}+\frac{2 n x_{i} x_{j}}{r^{2}\left(r-r_{1}\right)^{3}}-\frac{n\left(r^{2} \delta_{i j}-x_{i} x_{j}\right)}{r^{3}\left(r-r_{1}\right)^{2}}\right) .
$$


It follows from this and (2.1) that for some constant $\beta=\beta(n)$,

$$
\Delta V+\sum_{i, j} c_{i, j} V_{x_{i} x_{j}}+\sum_{i} c_{i} V_{x_{i}} \geq 0
$$

as long as $\left|c_{i, j}\right|,\left|c_{i}\right| \leq \beta$.

Fix a smooth non-decreasing function $a(r):[1,2] \rightarrow[0,1]$ with $a(1)=0$ and $a(2)=1$ and with $\left\{r \mid a^{\prime}(r)>0\right\}=\left(r_{0}, r_{1}\right)$. Let $b(r)=1-\kappa a(r)$ for some constant $\kappa \in(0,1)$ to be determined. Treating $b$ as a rotationally symmetric function of $x_{1}, \ldots, x_{n}$ consider the map $\left(y_{1}, \ldots, y_{n}\right)=\left(x_{1} / b, x_{2}, \ldots, x_{n}\right)$, which we will write as $y=\Psi(x)$. Note that $\Psi$ is invertible and $\Psi(x) \rightarrow \mathrm{Id}$ as $\kappa \rightarrow 0$ where the convergence is uniform in any $C^{k}$ norm on $\bar{\Omega}$. It follows from the inverse function theorem that we likewise have $\left.\Psi^{-1}(y)\right|_{\bar{\Omega}} \rightarrow \mathrm{Id}$ uniformly in any $C^{k}$ norm on $\bar{\Omega}$ as $\kappa \rightarrow 0$.

The map $\Psi(x)$ is the identity on $\left\{1 \leq r \leq r_{0}\right\}$ and takes the sphere $x_{1}^{2}+\cdots+x_{n}^{2}=R^{2}$ to the ellipsoid $b(R)^{2} y_{1}^{2}+y_{2}^{2}+\cdots+y_{n}^{2}=R^{2}$ which is non-spherical exactly when $R \in$ $\left(r_{0}, 2\right]$. We choose $\kappa$ sufficiently small so that the ellipsoid $(1-\kappa)^{2} y_{1}^{2}+y_{2}^{2}+\cdots+y_{n}^{2}=\left(r_{1}\right)^{2}$ is contained inside the sphere of radius 2 .

Define $W: \bar{\Omega} \rightarrow \mathbb{R}$ by $W(y)=V(x(y))$ for $x(y)=\Psi^{-1}(y)$. Note that $W=0$ outside the ellipsoid $(1-\kappa)^{2} y_{1}^{2}+y_{2}^{2}+\cdots+y_{n}^{2}=\left(r_{1}\right)^{2}$. Thus $W(y)$ satisfies (i), (ii) and (iii) of the Lemma.

To see that $y \cdot(\nabla W)(y) \leq 0$ we compute,

$$
y \cdot(\nabla W)(y)=\sum_{i, j} y_{i} V_{x_{j}}(x(y)) \frac{\partial x_{j}}{\partial y_{i}}=-(1+E) \frac{n r}{\left(r-r_{1}\right)^{2}}(x(y)) V(x(y))
$$

where $E$ is an "error" term which converges uniformly to zero as $\kappa$ tends to zero. Hence $y \cdot \nabla W \leq 0$ for $\kappa$ sufficiently small.

All that remains is to show now is that $\Delta W \geq 0$ in $\Omega$. We compute

$$
\begin{aligned}
(\Delta W)(y) & =\sum_{i, j, k} V_{x_{i} x_{j}}(x(y)) \frac{\partial x_{i}}{\partial y_{k}} \frac{\partial x_{j}}{\partial y_{k}}+\sum_{i, k} V_{x_{i}}(x(y)) \frac{\partial^{2} x_{i}}{\partial y_{k}^{2}} \\
& =(\Delta V)(x(y))+\sum_{i, j} c_{i, j} V_{x_{i} x_{j}}(x(y))+\sum_{i} c_{i} V_{x_{i}}(x(y)),
\end{aligned}
$$

for $c_{i, j}$ and $c_{i}$ which converge uniformly to zero as $\kappa$ tends to zero. From (2.3) it follows that $\Delta W \geq 0$ for $\kappa$ sufficiently small.

Next we have an elementary lemma about radially symmetric subharmonic functions.

Lemma 2.3. Let $f=f(r)$ be a smooth radially symmetric function on $\Omega$ with $f(1)=1$, $f(2)=0, f_{r} \leq 0$ and $\Delta f \geq 0$. Fix $r_{0}, r_{1}$ with $1<r_{0}<r_{1}<2$. Then

$$
f\left(r_{1}\right) \leq(1-\sigma) f\left(r_{0}\right),
$$

for $\sigma=\left(r_{1}-r_{0}\right) /\left(2+r_{1}-2 r_{0}\right)>0$.

Proof. We begin by showing

$$
-f_{r} \geq \frac{1}{2-r_{0}} f, \quad \text { on }\left[r_{0}, r_{1}\right] .
$$


Indeed the condition $\Delta f \geq 0$ implies that $r^{n-1}\left(-f_{r}(r)\right)$ is nonincreasing in $r$. Hence for $s \in\left[r_{0}, r_{1}\right]$ we have

$$
(2-s) s^{n-1}\left(-f_{r}(s)\right) \geq \int_{s}^{2} r^{n-1}\left(-f_{r}(r)\right) d r \geq s^{n-1} \int_{s}^{2}\left(-f_{r}(r)\right) d r=s^{n-1} f(s),
$$

where for the final equality we used $f(2)=0$, and (2.4) follows.

Next compute

$$
f\left(r_{0}\right)-f\left(r_{1}\right)=\int_{r_{0}}^{r_{1}}\left(-f_{r}(r)\right) d r \geq \frac{1}{2-r_{0}} \int_{r_{0}}^{r_{1}} f(r) d r \geq \frac{\left(r_{1}-r_{0}\right)}{2-r_{0}} f\left(r_{1}\right),
$$

where we recall for the last inequality that $f$ is decreasing in $r$. The result follows.

We can now complete the proof of Theorem 1.1

Proof of Theorem 1.1. Let $V=V_{5 / 4}$ be as in Lemma 2.1. Let $W$ be as in Lemma 2.2 with $r_{0}=5 / 4$ and $r_{1}=3 / 2$. We will write $v(t)$ and $w(t)$ for the solutions to (1.2) with initial conditions $V$ and $W$ respectively. For $\varepsilon \in(0,1)$ to be determined, define

$$
u_{0}=(1-\varepsilon) V+\varepsilon W,
$$

so that $u(t)=(1-\varepsilon) v(t)+\varepsilon w(t)$ is the solution of (1.2) starting at $u_{0}$. Clearly $u_{0}$ is an admissible function. In addition, note that for $\{1<r \leq 5 / 4\}$ the level sets of $u_{0}$ are spheres and, since $V$ vanishes for $r>5 / 4$, the level sets for $u_{0}$ on $\{r>5 / 4\}$ are the same as those of $W$. In particular, the level sets of $u_{0}$ are convex and so $u_{0}$ is quasiconcave.

Let $\eta_{1}, \eta_{2} \in(0,1 / 4)$ be small constants to be determined and write $R^{-}=3 / 2-$ $\eta_{1}$. Then on the non-spherical ellipsoid $E_{R^{-}}$with equation given by (2.2), $W$ takes a constant value, $W=s>0$, say, which depends on $\eta_{1}$. We have $W=0$ on and outside the ellipsoid $E_{3 / 2}$. Define $R^{+}=3 / 2+\eta_{2}$ and write $S_{R^{+}}$for the sphere of radius $R^{+}$. Observe that for $\eta_{2}$ sufficiently small we can find $X \in S_{R^{+}}$and $Y^{\prime} \in E_{3 / 2}$ such that $\left(X+Y^{\prime}\right) / 2$ lies outside both $S_{R^{+}}$and $E_{3 / 2}$. Next by choosing $\eta_{1}$ sufficiently small we can find a point $Y \in E_{R^{-}}$close to $Y^{\prime}$ so that $Z=(X+Y) / 2$ lies outside both $S_{R^{+}}$and $E_{3 / 2}$. See Figure 1, Note that now $\eta_{1}$ is chosen, $s$ is a fixed positive number.

Since $V(X)=W(Z)=0$, and using Proposition 2.1, there exist continuous functions $\alpha(t), \beta(t)$ which vanish at $t=0$ and are positive for $t>0$ such that

$$
v(X, t)=s \alpha(t), \quad w(Z, t)=\beta(t) .
$$

Next we use Lemma 2.3 to see that there exists a constant $\sigma>0$ independent of $t$ such that

$$
v(Z, t) \leq s(1-\sigma) \alpha(t)
$$

Indeed, the radially symmetric function $v(\cdot, t)$ satisfies the conditions of Lemma 2.3 and $Z$ lies at a fixed distance outside the sphere $S_{R^{+}}$which contains $X$.

Next note that since $W(Y)=s$ and $w_{t} \geq 0$, we have

$$
w(Y, t) \geq s
$$

for all $t \geq 0$. Choose a small time $t_{0}>0$ such that

$$
\varepsilon:=\alpha\left(t_{0}\right)<1 / 2, \quad \text { and } \beta\left(t_{0}\right)<\frac{\sigma s}{2} .
$$




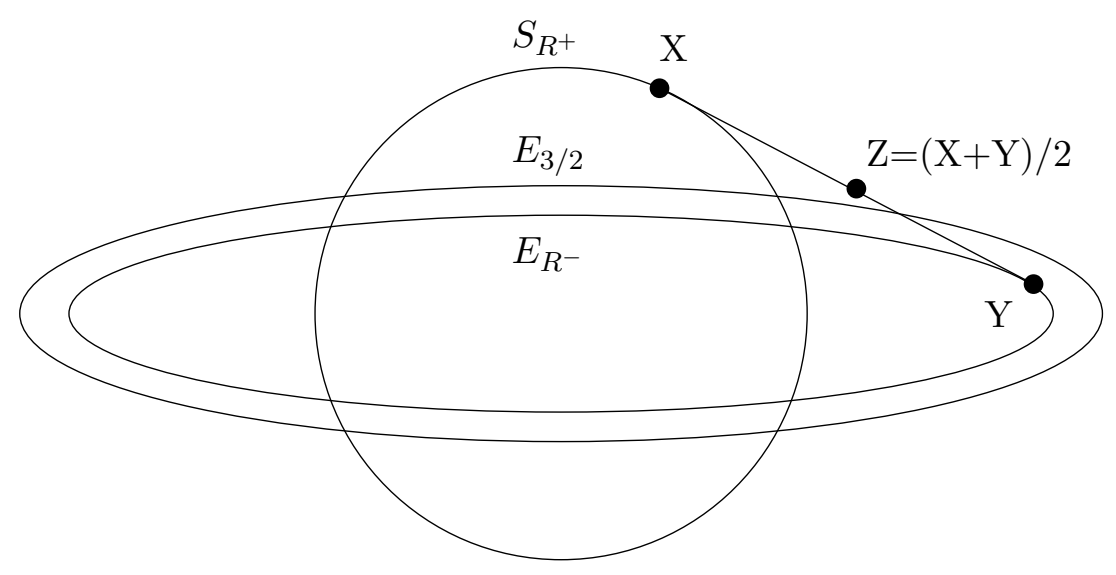

FigurE 1. Schematic diagram showing the ellipsoids $E_{R^{-}}$and $E_{3 / 2}$, the sphere $S_{R^{+}}$and the points $X, Y, Z$.

Compute that

$$
u\left(Z, t_{0}\right) \leq(1-\varepsilon) s(1-\sigma) \varepsilon+\frac{\varepsilon \sigma s}{2}<(1-\varepsilon) s \varepsilon
$$

But

$$
u\left(X, t_{0}\right) \geq(1-\varepsilon) s \varepsilon, \quad u\left(Y, t_{0}\right) \geq \varepsilon s>(1-\varepsilon) s \varepsilon .
$$

Hence $X$ and $Y$ lie in the superlevel set $\left\{P \mid u\left(P, t_{0}\right) \geq(1-\varepsilon) s \varepsilon\right\}$ but $Z=(X+Y) / 2$ does not, showing that this superlevel set is not convex.

\section{A RADially Symmetric EXAMPLE}

In this section we give the proof of Theorem 1.2, First, define a smooth function $h:[0,1] \rightarrow \mathbb{R}$ to have the following properties

(a) $1 / 10 \geq h(r)>0$ for $r \in(0,1)$.

(b) $h(r)=1 / 10$ for $r \in[1 / 4,3 / 4]$.

(c) $h^{(k)}(0)=0=h^{(k)}(1)$ for all $k=0,1,2, \ldots$

Next, for a constant $R>1$, define $v_{R}:[0,1] \rightarrow \mathbb{R}$ to be the solution of the Dirichlet problem

$$
v_{R}^{\prime \prime}(r)+(n-1) \frac{v_{R}^{\prime}(r)}{r+R}=h(r), 0<r<1, \quad v_{R}(0)=1, v_{R}(1)=0 .
$$

We will determine the constant $R$ later. By standard elliptic estimates [18] we have that $v_{R}(r)$ and its derivatives are bounded on $[0,1]$ uniformly with respect to $R>1$.

Remark 3.1. In fact in what follows we only need that $\left|v_{R}^{\prime}(r)\right| \leq C$ for a constant $C$ independent of $R$. In this case we can actually write down the solution $v_{R}$ explicitly and prove this directly. For example, when $n=2, v_{R}(r)$ is given by

$$
v_{R}(r)=\int_{0}^{r} \frac{1}{x+R}\left(\int_{0}^{x}(y+R) h(y) d y\right) d x+c_{1} \log (r+R)+c_{2}
$$


for constants $c_{1}$ and $c_{2}$ given by

$$
c_{1}=\frac{-1-\int_{0}^{1} \frac{1}{x+R}\left(\int_{0}^{x}(y+R) h(y) d y\right) d x}{\log ((1+R) / R)}, \quad c_{2}=1-c_{1} \log R .
$$

To see that $\left|v_{R}^{\prime}(r)\right| \leq C$ for a constant $C$ independent of $R$, note that as $R \rightarrow \infty$ the term

remains bounded.

$$
\left|\frac{c_{1}}{r+R}\right|=\mathrm{O}\left(\frac{1 / R}{\log \left(1+\frac{1}{R}\right)}\right)
$$

We can now start the proof of Theorem 1.2 .

Proof of Theorem 1.2. Define our radially symmetric function $u_{0}$ on $\bar{\Omega}$ by

$$
u_{0}(r)=v_{R}(r-R), \quad \text { for } R \leq r \leq R+1,
$$

which satisfies the boundary conditions $u_{0}(R)=1, u_{0}(R+1)=0$. Moreover, from (3.1) we have

$$
\Delta u_{0}(r)=h(r-R), \quad \text { for } R \leq r \leq R+1 .
$$

Now notice that by the definition of $h$ we have for $r=R$ or $r=R+1$,

$$
\Delta^{k} u_{0}(r)=0, \quad \text { for every } k \geq 1 \text {. }
$$

Let $u(r, t)$ be the rotationally symmetric solution to (1.2) on $\Omega$ with initial condition $u_{0}$. Then from [28, Theorem 5.2] (or [27, Theorem 10.4.1]) it follows that the function $u(r, t)$ extends to a smooth function on $\bar{\Omega} \times[0, \infty)$.

Next we show that for sufficiently large $R$, the function $u_{0}$ satisfies the hypotheses of Proposition 2.1.

Lemma 3.1. For sufficiently large $R$ we have

(i) $u_{0}(r)$ is strictly decreasing in $r$.

(ii) $u_{0}^{\prime \prime}(R+1 / 2) \geq 1 / 10$.

(iii) $\Delta u_{0}>0$ for $R<r<R+1$.

Proof. For (i), note that for all $r \in[R, R+1]$ we have $\left|u_{0}^{\prime}(r)\right| \leq C$ for some $\mathrm{C}$ independent of $R$ as observed above. Thus for all $r \in[R, R+1]$ we have

$$
\begin{aligned}
\left|u_{0}^{\prime \prime}(r)\right| & \leq\left|\Delta u_{0}(r)\right|+(n-1)\left|u_{0}^{\prime}(r) / r\right| \\
& =|h(r-R)|+(n-1)\left|u_{0}^{\prime}(r) / r\right| \\
& \leq 1 / 10+(n-1) C / R \leq 1 / 5
\end{aligned}
$$

as long as $R$ is larger than $10 C(n-1)$. On the other hand, by the boundary conditions on $u_{0}$ we have by the Mean Value Theorem that $u_{0}^{\prime}\left(r_{0}\right)=-1$ at some point $r_{0}$ in $[R, R+1]$. Hence $u_{0}^{\prime}(r) \leq-1+1 / 5$ for all $r \in[R, R+1]$, giving (i).

For (ii), we have

$$
\begin{aligned}
u_{0}^{\prime \prime}(R+1 / 2) & =\Delta u_{0}(R+1 / 2)-(n-1) u_{0}^{\prime}(R+1 / 2) /(R+1 / 2) \\
& \geq \Delta u_{0}(R+1 / 2) \\
& =h(1 / 2)=1 / 10
\end{aligned}
$$


where in the second inequality we have used part (i) and in the third inequality we have used the definition of $u_{0}$ and the property (b) of $h$.

Finally (iii) follows from the definition of $h$.

We now fix $R$ as in the lemma above. Define $c=u_{0}(R+1 / 2)$ and let $u(x, t)$ be the solution to (1.2). We consider the space-time superlevel set

$$
\Omega_{c}:=\{(x, t) \in \bar{\Omega} \times[0, \infty): u(x, t) \geq c\} \cup\left(\Omega_{1} \times[0, \infty)\right) .
$$

As noted above, $u(x, t)$ is smooth on $\bar{\Omega} \times[0, \infty)$ while from Lemma 3.1 and Proposition 2.1. $u_{t}(x, t)$ is smooth and strictly positive on $\Omega \times[0, \infty)$. By the Implicit Function Theorem, we may write $\partial \Omega_{c}=\{(x, t) \in \bar{\Omega} \times[0, \infty): u(x, t)=c\}$ as the radial graph of the equation $t=f(r)$ where $u(r, f(r))=c$ and $f(r)$ is a smooth increasing function on $[R+1 / 2, R+1 / 2+\varepsilon)$ for some $\varepsilon>0$. Note that $f(R+1 / 2)=0$. Differentiating the defining equation for $f$ we obtain

$$
f^{\prime}(r)=-\frac{u_{r}}{u_{t}}
$$

where the functions above are evaluated at $(r, f(r))$, and

$$
f^{\prime \prime}(r)=\frac{-1}{u_{t}}\left(u_{r r}+2 u_{r t} f^{\prime}+u_{t t}\left(f^{\prime}\right)^{2}\right)
$$

where again these are all evaluated at $(r, f(r))$. Now evaluating the above at $(r, f(r))=$ $(R+1 / 2,0)$, and noting that $u_{t}(r, 0)=\Delta u_{0}(r)=h(r-R)=1 / 10$ in some neighborhood of $r=R+1 / 2$, hence $u_{t r}(R+1 / 2,0)=u_{t t}(R+1 / 2,0)=0$, we get

$$
f^{\prime \prime}(R+1 / 2)=-10 u_{0}^{\prime \prime}(R+1 / 2) \leq-1<0
$$

by Lemma 3.1. This contradicts that $\partial \Omega_{c}$, which is defined by $t=f(r)$, is convex in $\mathbb{R}^{n+1}$.

\section{Two-Point FunCtions}

In this section we discuss a different way to find a counterexample to space-time quasiconcavity using a two-point function as in [35. (see also [30]). We work in dimension $n \geq 1$ with the domain $\Omega=\{1<r<2\}$. Let $u(x, t)$ be a smooth function on $\bar{\Omega} \times[0, \infty)$. Consider the two-point function

$$
\mathcal{H}((x, s),(y, t))=(D u(y, t)-D u(x, s)) \cdot(y-x)+\left(u_{t}(y, t)-u_{t}(x, s)\right)(t-s),
$$

restricted to $(x, s),(y, t) \in \bar{\Omega} \times[0, \infty)$ with $u(x, s)=u(y, t)$. If $u(x, t)$ is space-time quasiconcave then $\mathcal{H} \leq 0$. Indeed, note that for a smooth function $w$ in $\mathbb{R}^{n+1}$, if the superlevel set $\{w>c\}$ is convex with a smooth boundary that contains two points $X$ and $Y$ then $(D w(Y)-D w(X)) \cdot(Y-X) \leq 0$ since the vectors $D w(X), D w(Y)$ point in the inward normal direction. Applying this to $X=(x, s)$ and $Y=(y, t)$ in $\bar{\Omega} \times(0, \infty)$ with $u(x, s)=u(y, t)$, and using the continuity of $\mathcal{H}$, we have $\mathcal{H} \leq 0$ on its domain of definition.

For our counterexample, we construct a radially symmetric admissible function $u_{0}$ such that the corresponding solution $u(x, t)$ of the heat equation (1.2) is smooth on $\bar{\Omega} \times[0, \infty)$ and has $\mathcal{H}$ strictly positive somewhere.

Let $\varepsilon>0$ be a small constant to be determined and let $g:[1,2] \rightarrow[0, \infty)$ be a smooth function with the following properties: 
(a) $g(r)=1 /(2 \varepsilon)$ for $1 \leq r \leq 1+\varepsilon$, and $g(r)=0$ for $2-\varepsilon \leq r \leq 2$.

(b) $g$ is decreasing on $[1,2]$.

(c) $\int_{1}^{2} r^{1-n} g(r) d r=1$.

We then define a smooth function $u_{0}$ on $[1,2]$ by

$$
u_{0}(r)=-\int_{1}^{r} s^{1-n} g(s) d s+1, \quad r \in[1,2] .
$$

It is straightforward to check that $u_{0}$ is an admissible function. Moreover, $\Delta u_{0}$ vanishes identically in a neighborhood of $r=1$ and $r=2$. Let $u(x, t)$ be the corresponding solution of (1.2), which by [28, Theorem 5.2] is smooth on $\bar{\Omega} \times[0, \infty)$. From Proposition 2.1 we know that $u(x, t)$ converges smoothly as $t \rightarrow \infty$ to the harmonic function $u_{\infty}$ given by

$$
u_{\infty}(r)= \begin{cases}2-r, & n=1 \\ 1-\frac{\log r}{\log 2}, & n=2 \\ \frac{(2 / r)^{n-2}-1}{2^{n-2}-1}, & n>2 .\end{cases}
$$

We now choose our points $(x, s)$ and $(y, t)$. We choose $x$ and $y$ to lie in the line $x_{2}=\cdots=x_{n}=0$. Pick $x=(1+\varepsilon / 2,0, \ldots, 0)$ and $s=0$. By the definition of $u_{0}$ we have $u(x, 0)=u_{0}(1+\varepsilon / 2) \approx 3 / 4$. Let $\gamma$ solve $u_{\infty}(1+\gamma)=u(x, 0)$, which satisfies $\gamma>c(n)$ for a constant $c(n) \in(0,1)$ depending only on $n$. Writing $y(t)=\left(y_{1}(t), 0, \ldots, 0\right)$ solving $u(y(t), t)=u(x, 0)$ we have $y_{1}(t) \rightarrow 1+\gamma$ and $u_{r}(y(t), t) \rightarrow\left(u_{\infty}\right)_{r}(1+\gamma)$ as $t \rightarrow \infty$. Then for $t$ sufficiently large and $\varepsilon>0$ sufficiently small we have $y_{1}>1+\varepsilon / 2$ and $\left|u_{r}(y(t), t)\right| \leq C$ for a uniform $C$. Writing $y$ for $y(t)$ we have

$$
\mathcal{H}((x, s),(y, t))=\left(u_{r}(y, t)-u_{r}(x, 0)\right)\left(y_{1}-(1+\varepsilon / 2)\right)+\left(u_{t}(y, t)-u_{t}(x, 0)\right)(t-0) .
$$

From the definition of $u_{0}$ we have $u_{r}(x, 0) \approx-1 /(2 \varepsilon)$ and $u_{t}(x, 0)=\Delta u(x, 0)=0$. On the other hand, from Proposition 2.1, $u_{t}(y, t)>0$. Hence for $\varepsilon$ sufficiently small, $\mathcal{H}>0$.

Remark 4.1. As in [35] one can show that a maximum principle holds for the quantity $\mathcal{H}$ using a parabolic version of a Lemma of Rosay-Rudin [30]. This rules out $\mathcal{H}$ obtaining a positive interior maximum. However, it does not rule out a positive maximum occuring at a point $((x, s),(y, t))$ with $s=0$ which would be needed to prove that quasiconcavity is preserved for the heat equation.

\section{REFERENCES}

1. Ahlfors, L.V., Conformal invariants: Topics in geometric function theory, McGraw-Hill Series in Higher Mathematics. McGraw-Hill Book Co., New York-Düsseldorf-Johannesburg, 1973.

2. Alvarez, O., Lasry, J.-M., Lions, P.-L., Convex viscosity solutions and state constraints, J. Math. Pures Appl. (9) 76 (1997), no. 3, 265-288.

3. Bian, B., Guan, P., A microscopic convexity principle for nonlinear partial differential equations, Invent. Math. 177 (2009), 307-335.

4. Bian, B., Guan, P., Ma, X.N., Xu, L., A constant rank theorem for quasiconcave solutions of fully nonlinear partial differential equations, Indiana Univ. Math. J. 60 (2011), no. 1, 101-119.

5. Bianchini, C., Longinetti, M., Salani, P., Quasiconcave solutions to elliptic problems in convex rings, Indiana Univ. Math. J. 58 (2009), no. 4, 1565-1589.

6. Borell, C., Brownian motion in a convex ring and quasiconcavity, Comm. Math. Phys. 86 (1982), no. $1,143-147$. 
7. Brascamp, H.J., Lieb, E.H. On extensions of the Brunn-Minkowski and Prékopa-Leindler theorems, including inequalities for log concave functions, and with an application to the diffusion equation, J. Functional Analysis 22 (1976), no. 4, 366-389.

8. Caffarelli, L., Friedman, A., Convexity of solutions of some semilinear elliptic equations, Duke Math. J. 52 (1985), no. 2, 431-456.

9. Caffarelli, L., Guan, P., Ma, X.N., A constant rank theorem for solutions of fully nonlinear elliptic equations, Comm. Pure Appl. Math. 60 (2007), no. 12, 1769-1791.

10. Caffarelli, L., Spruck, J., Convexity properties of solutions to some classical variational problems, Comm. Partial Differential Equations 7 (1982), no. 11, 1337-1379.

11. Chang, S.-Y.A., Ma, X.-N., Yang, P., Principal curvature estimates for the convex level sets of semilinear elliptic equations, Discrete Contin. Dyn. Syst. 28 (2010), no. 3, 1151-1164.

12. Chen, C.Q., Hu, B.W., A Microscopic Convexity Principle for Space-time Convex Solutions of Fully Nonlinear Parabolic Equations, Acta Math. Sin. (English Ser.) 29 (2013), no. 4, 651-674.

13. Chen, C.Q., Shi, S.J. Curvature estimates for the level sets of spatial quasiconcave solutions to a class of parabolic equations, Science China Mathematics 54 (2011), no. 10, 2063-2080.

14. Diaz, J.I., Kawohl, B., Convexity and starshapedness of level sets for some nonlinear parabolic problems., in Free boundary problems: theory and applications, Vol. II (Irsee, 1987), 883-887, Pitman Res. Notes Math. Ser., 186, Longman Sci. Tech., Harlow, 1990.

15. Diaz, J.I., Kawohl, B., On convexity and starshapedness of level sets for some nonlinear elliptic and parabolic problems on convex rings, J. Math. Anal. Appl. 177 (1993), no. 1, 263-286.

16. Friedman, A., Partial differential equations of parabolic type, Prentice-Hall, Inc., Englewood Cliffs, N.J. 1964.

17. Gabriel, R., A result concerning convex level surfaces of 3-dimensional harmonic functions, J. London Math. Soc. 32 (1957), 286-294.

18. Gilbarg, D., Trudinger, N.S., Elliptic partial differential equations of second order, Springer-Verlag, Berlin, 2001.

19. Hamel, F., Nadirashvili, N., Sire, Y., Convexity of level sets for elliptic problems in convex domains or convex rings: two counterexamples, Amer. J. Math. 138 (2016), no. 2, 499-527.

20. Hu, B.W., Ma, X.N., Constant rank theorem of the spacetime convex solutions of heat equation, Manuscripta Math., 138 (2012), no. 1-2, 89-118.

21. Ishige, K., Salani, P., Is quasi-concavity preserved by heat flow?, Arch. Math. (Basel) 90 (2008), no. $5,450-460$.

22. Ishige, K., Salani, P., Parabolic quasi-concavity for solutions to parabolic problems in convex rings, Math. Nachr. 283 (2010), no. 11, 1526-1548.

23. Ishige, K., Salani, P., On a new kind of convexity for solutions of parabolic problems, Discrete Contin. Dyn. Syst. Ser. S 4 (2011), no. 4, 851-864.

24. Kawohl, B., Rearrangements and convexity of level sets in PDE. Lecture Notes in Mathematics, 1150. Springer-Verlag, Berlin, 1985.

25. Korevaar, N.J., Convexity of level sets for solutions to elliptic ring problems, Comm. Partial Differential Equations 15 (1990), no. 4, 541-556.

26. Korevaar, N.J., Lewis, J.L., Convex solutions of certain elliptic equations have constant rank Hessians, Arch. Rational Mech. Anal. 97 (1987), no. 1, 19-32.

27. Krylov, N.V., Lectures on elliptic and parabolic equations in Hölder spaces. Graduate Studies in Mathematics, 12. American Mathematical Society, Providence, RI, 1996.

28. Ladyženskaja, O.A., Solonnikov, V.A., Ural'ceva, N.N., Linear and quasilinear equations of parabolic type, Translations of Mathematical Monographs, Vol. 23 American Mathematical Society, Providence, RI, 1968.

29. Lewis, J., Capacitary functions in convex rings, Arch. Rational Mech. Anal. 66 (1977), 201-224.

30. Rosay, J.-P., Rudin, W., A maximum principle for sums of subharmonic functions, and the convexity of level sets, Michigan Math. J. 36 (1989), no. 1, 95-111.

31. Shiffman, M., On surfaces of stationary area bounded by two circles or convex curves in parallel planes, Ann. of Math. (2) 63 (1956), 77-90.

32. Singer, I., Wong, B., Yau, S.T., Yau, S.S.T., An estimate of gap of the first two eigenvalues in the Schrodinger operator, Ann. Scuola Norm. Sup. Pisa Cl. Sci. (4) 12 (1985), no. 2, 319-333. 
33. Székelyhidi, G., Weinkove, B., On a constant rank theorem for nonlinear elliptic PDEs, Discrete Contin. Dyn. Syst. 36 (2016), no. 11, 6523-6532.

34. Wang, X.-J., Counterexample to the convexity of level sets of solutions to the mean curvature equation, J. Eur. Math. Soc. (JEMS) 16 (2014), no. 6, 1173-1182.

35. Weinkove, B., Convexity of level sets and a two-point function, to appear in Pacific J. Math., arXiv:1701.05820

Department of Mathematics, The University of British Columbia, 1984 Mathematics Road, Vancouver, B.C., Canada V6T 1Z2. Email: Chau@math.ubc.ca.

Department of Mathematics, Northwestern University, 2033 Sheridan Road, Evanston, IL 60208, USA. EMAIL: WEINKOVE@MATH.NORTHWESTERN.EDU. 